\title{
АНАЛИЗ С-ГЛИКОЗИДОВ ФЛАВОНОВ И ПРОДУКТОВ СТУПЕНЧАТОГО ГИДРОЛИЗА ИХ АЦЕТАТОВ В ЛИСТЬЯХ RUBUS CHAMAEMORUS L.*
}

\author{
(C) А.К. Уэйли', А.О. Понкратова ${ }^{1 * *}$, А.А. Орлова ${ }^{1}$, Е.Б. Серебряков ${ }^{2}$, С.И. Селиванов ${ }^{2}$, \\ С.В. Кривощеков ${ }^{3}$ М.В. Белоусов ${ }^{3}$, П. Проки ${ }^{4}$, В.Г. Лужанин ${ }^{1}$ \\ ${ }^{1}$ Санкт-Петербургский государственный химико-фрармацевтический \\ университет, ул. Проф. Попова 14, Санкт-Петербург, 197376 (Россия), \\ e-mail: anastasiya.ponkratova@yandex.ru \\ ${ }^{2}$ Санкт-Петербургский государственный университет, \\ Университетский пр., 26, Санкт-Петербург, 198504 (Россия) \\ ${ }^{3}$ Сибирский государственный медицинский университет, \\ Московский тракт, 2, Томск, 634050 (Россия) \\ ${ }^{4}$ Institute of Pharmaceutical Biology and Biotechnology, Heinrich-Heine-Universität \\ Düsseldorf, Universitätsstrasse 1, Düsseldorf, 40225 (Germany)
}

С-гликозид эмбинина и его моно- и диацетатные производные обладают иммунотропной и кардиотонической активностью, что определяет перспективность поиска их растительных источников. Эмбинин и его ацетатные производные ранее выделялись только из некоторых растений рода Iris, ареал и условия произрастания которых сильно отличается от растений рода Rubus. В результате проведенного исследования установлена структура семи выделенных из листьев Rubus chamaemorus L. (Rosaceae) С-гликозидов - производных эмбинина (1). Методами HR-ESI-MS, BЭЖX-MC, а также одно- и двумерной ЯМР-спектроскопии установлена структура трех веществ выделенных в индивидуальном виде: эмбинин (1) и его диацетил производных - 2"',3"'-диацетилэмбинин (5) и $3 "$ "',4"'-диацетилэмбинин (7). Предложенный в данном исследовании метод ступенчатого гидролиза ацетатных остатков С-гликозидов с последующим ВЭЖХанализом образующихся продуктов гидролиза позволил установить структуру минорных С-гликозидов флавонов, содержащихся в листьях Rubus chamaemorus L.: 2"'-ацетилэмбинин (2), 3"'-ацетилэмбинин (3), 4"'-ацетилэмбинин (4) и 2"',4"'-диацетилэмбинин (6). Все указанные соединения обнаружены в листьях Rubus chamaemorus L. впервые. C-гликозиды - эмбинин и его ацетатные производные представляют собой редкие метаболиты высших растений, наличие которых определяется особенностью их физиологии, а биологическая активность обусловливает перспективность медицинского применения.

Ключевые слова: С-гликозиды, флавоны, гидролиз, морошка обыкновенная, вторичные метаболиты.

\section{Введение}

Rubus chamaemorus L. (морошка обыкновенная) - двудомное многолетнее травянистое растение из семейства розоцветных (Rosaceae), произрастающее преимущественно по всему северному полушарию, включая Северную Европу, Россию и Северную Америку [1-3]. Фитохимический анализ морошки до этого момента был в основном сосредоточен на ягодах растения, из-за их пищевой ценности и применения в пищевой промышленности [4]. Основная группа вторичных метаболитов, содержащихся в плодах $R$. chamaemorus, представляет собой полифенолы, среди которых гидролизуемые таннины могут рассматри-

Уэйли Андрей Кеннет - аспирант, младший научный сотрудник, e-mail: 9968639@gmail.com

Понкратова Анастасия Олеговна - аспирант, младший научный сотрудник, e-mail: anastasiya.ponkratova@yandex.ru

Орлова Анастасия Андреевна - аспирант, e-mail: anastasiya.lebedkova@spcpu.ru

Окончание на С. 258. ваться в качестве мажорных компонентов [5-8]. Известно, что в морошке содержатся флавоноиды и О-гликозиды флавоноидов [9-11]. При этом листья морошки, являясь при сборе ягоды доступным потенциальным сырьевым источником, практически не изучены.

\footnotetext{
*Данная статья имеет электронный дополнительный материал (приложение), который доступен читателям на сайте журнала. DOI: 10.14258/jcprm.2021029185s

** Автор, с которым следует вести переписку.
} 
McDougall с соавт. при анализе спелых ягод $R$. chamaemorus с помощью нового масс-спектрометрического метода идентифицировали сигнал в полученном масс-спектре положительной ионизации с $m / z 607$ (идентичный эмбинину) и, используя данные $\mathrm{MC/MC}$, предложили структуру - кверцетин 3-О-[6"-(3-гидрокси-3метилглутароил)- $\beta$-глюкозид] [5]. Другие авторы, использующие метод ВЭЖХ-МС/МС для анализа плодов морошки, наблюдали неидентифицированный ион с $m / z$ 695, дающий дочерние ионы при фрагментации с $m / z$ 649 и 487 [9]. Установлено, что данный ион с $m / z 695$ представляет собой аддукт молекулярного иона с муравьиной кислотой типа $[(\mathrm{M}+\mathrm{H})+\mathrm{HCOOH}]^{+}$, используемый авторами работы [9] в качестве добавки к подвижной фазе. При регистрации МС/MC-спектров в нейтральной среде регистрируется сигнал истинного молекулярного иона $[\mathrm{M}+\mathrm{H}]^{+} m / z 649$, который может быть отнесен к моноацетатному производному эмбинина. Дальнейшая фрагментация приводит к образованию иона с $m / z 487$, что характерно для эмбинина.

Поскольку С-гликозиды флавона - эмбинин и его производные ранее выделялись только из некоторых представителей рода Iris [12-17], а исследования биологической активности эмбинина показали, что данное соединение обладает выраженной иммунотропной и кардиотонической активностью [18, 19], то данные об их обнаружении у представителей рода Rubus являются оригинальными и могут обладать ценностью как в области физиологии, биохимии и систематики растений, выступая в качестве уникальных метаболических и хемотаксономических маркеров, так и для наук фармацевтической отрасли, выявляя новый потенциальный источник лекарственного сырья.

\section{Материалы и методы}

Листья R. chamaemorus собраны в Ленинградской области, вблизи Питомника лекарственных растений Санкт-Петербургского государственного химико-фармацевтического университета (Ленинградская область, Всеволожский район, 38 км приморского шоссе). Изучаемое сырье морфологически соответствует гербарному образцу вида $R$. chamaemorus, хранящегося на кафедре фармакогнозии СПХФУ (№ SPCPU19RC, собрал и определил д.б.н. Гончаров М.Ю., август 2017 года). Собранные листья перед транспортировкой высушивали в инфракрасной сушилке при $40{ }^{\circ} \mathrm{C}$. Перед экстракцией листья измельчали и просеивали через сито с диаметром отверстий 1 мм. Навеску сырья (700 г) многократно экстрагировали 96\% этанолом в соотношении $1: 8$ при комнатной температуре до обесцвечивания получаемого экстракта. Полученное этанольное извлечение упаривали в вакуумно-ротационном испарителе (Heidolph, Германия) в 15 раз, после чего к концентрату добавляли воду $(10: 1)$. Далее проводили жидкость-жидкостную экстракцию $н$-гексаном (в соотношении $1: 1$ до обесцвечивания н-гексановой фазы). После чего к водно-спиртовому остатку прибавляли 400 мл воды и проводили экстракцию дихлорметаном в соотношении $1: 1$. Оставшийся водный остаток упаривали на вакуумно-ротационном испарителе при $60^{\circ} \mathrm{C}$ до $1 / 5$ исходного объема и наносили на колонку, заполненную сорбентом Dianion HР-20. Проводили градиентное элюирование со скоростью 10 мл/мин от 100\% воды до 100\% EtOH с шагом в 10\%, объем каждой порции элюента составляла 1 л. Фракцию, элюирующуюся с колонки 40\% этанолом, собирали, упаривали и перерастворяли в минимальном объеме метанола. Переносили на колонку с сорбентом Sephadex LH-20 и хроматографировали изократически с использованием метанола. Подфракции собирали в 10 мл пробирки, которые затем объединяли на основании их тонко-

Серебряков Евгений Борисович - директор ресурсного центра «Методы анализа состава веществ», e-mail: evgeny030403@yandex.ru

Селиванов Станислав Иванович - доктор химических наук, доцент, заведующий кафедрой химии природных соединений, e-mail: s.selivanov@spbu.ru

Кривощеков Сергей Владимирович - заведующий лабораторией контроля качества,

e-mail:ksv_tsu@mail.ru

Белоусов Михаил Валерьевич - доктор фармацевтических наук, заведующий кафедрой фармацевтического анализа, e-mail: mvb63@mail.ru

Прокш Петер - профессор, директор института фармацевтической биологии и биотехнологии, e-mail: proksch.juelich@t-online.de

Лужанин Владимир Геннадьевич - заведующий кафедрой фармакогнозии, проректор по воспитательной и административно-кадровой работе, e-mail: vladimir.luzhanin@pharminnotech.com слойных хроматограмм в системе БУВ 4:1:2. Объединенные подфракции анализировались методом ВЭЖХ с диодно-матричным детектором. Подфракции, содержащие пики с характерными для Cгликозидов флавонов УФ-спектрами поглощения, отбирались для дальнейшего выделения методом препаративной ВЭЖХ.

Препаративное разделение суммы гликозидов проводили на хроматографе Smartline (Knauer, Германия) со спектрофотометрическим детектором $(\lambda=270$ нм), снабженным колонкой Kromasil C18 $(250 \times 30$ мм, 5 мкм). Разделение проводили в градиентном режиме: выдержка при 5\% В - 5 мин, подъем до 50\% В - 35 мин, выдержка при 50\% - 5 мин, скорость потока 40 мл/мин (подвижная фаза А 
- 0.1\% (об./об.) ТФУ в воде; подвижная фаза В - 0.1\% (об./об.) ТФУ в ацетонитриле). Отдельные пики индивидуальных С-гликозидов собирались в момент их регистрации на детекторе в соответствующие каждому из них емкости.

Анализ собранных фракций проводили на жидкостном хроматографе Prominence LC-20 (Shimadzu, Япония) с колонкой Supelcosil C18 (250 × 4.6 мм, 5 мкм), оснащенным диодно-матричным детектором SPDM20A (регистрация спектров в диапазоне 200-800 нм). Разделение проводили в градиентном режиме: выдержка при 5\% В - 5 мин, подъем до 100\% В - 45.75 мин, выдержка при 100\% - 4.25 мин, скорость подвижной фазы составляла 1 мл/мин (подвижная фаза А - 0.1\% (об./об.) ТФУ в воде; подвижная фаза В - 0.1\% (об./об.) ТФУ в ацетонитриле).

Спектры HR-ESI-MS получены с использованием спектрометра Bruker Micromass Q-TOF (CША) peжим ионизации - электрораспыление; температура нагревательного блока - $300{ }^{\circ} \mathrm{C}$; скорость газа-распылителя (N2) - 3 л/мин; напряжение на капилляре - 3 кВ; диапазон сканирования масс $(m / z) 95-1500$ a.e.м. Спектры 1D и 2D ЯМР регистрировали на ЯМР-спектрометре Bruker DPX 300 МГц (США) в ДМСО- $d_{6}$ При температуре $25^{\circ} \mathrm{C}$ с привязкой к дейтериевому резонансу растворителя. Тандемную масс-спектрометрию проводили с использованием FLEXAR SQ 300 MS (PerkinElmer, США) режим ионизации - электрораспыление; температура интерфейса ИЭР - $300{ }^{\circ} \mathrm{C}$; температура нагревательного блока $-300{ }^{\circ} \mathrm{C}$; скорость газа-распылителя (N2) - 3 л/мин; напряжение на капилляре - 3 кВ; диапазон сканирования масс (m/z) 200-900 a.e.м.

\section{Результаты и обсуждение}

При элюировании метанолом с колонки, заполненной Sephadex LH-20, получена фракция, содержащая С-гликозиды (рис. 1). Данные соединения характеризуются практически идентичными спектрами УФпоглощения, с двумя характеристическими максимумами при 271 и 333 нм (или 334 нм), характерные для С-гликозидных производных апигенина [6].

С помощью разделения С-гликозидной фракции на обращено-фазовой препаративной колонке получено 7 индивидуальных компонентов (1-7) с хроматографической чистотой пиков от 95-99\%. Из них соединения 1, 5 и 7 выделены индивидуально в количестве, достаточном для определения структуры с использованием набора спектральных методов (5.2 мг, 10.4 и 14.7 мг, соответственно).

На спектре HR-ESI-MS соединения 1 наблюдали сигналы псевдомолекулярных ионов с $m / z$ 607.1994, 629.1823 и 645.1557, соответствующих $\left[\mathrm{C}_{29} \mathrm{H}_{34} \mathrm{O}_{14}+\mathrm{H}\right]^{+},\left[\mathrm{C}_{29} \mathrm{H}_{34} \mathrm{O}_{14}+\mathrm{Na}\right]^{+}$и $\left[\mathrm{C}_{29} \mathrm{H}_{34} \mathrm{O}_{14}+\mathrm{K}\right]^{+}$, однако соединения 5 и 7 не обладают этим свойством, на спектрах регистрируется сигнал с $\mathrm{m} / z$ 691.2157, что соответствует $\left[\mathrm{C}_{33} \mathrm{H}_{38} \mathrm{O}_{16}+\mathrm{H}\right]^{+}$.

Анализ одномерных ${ }^{1} \mathrm{H},{ }^{13} \mathrm{C}$, DEPT и двумерных COSY, NOESY, HSQC, HMBC ЯМР-экспериментов соединения 1, 5 и 7 показал, что все три выделенные соединения проявляли дублированные сигналы в диапазоне $\delta_{\mathrm{H}} 0.3-13.5$, которые относятся к мажорному и минорному ротамерам, возникающим из-за заторможенного вращения вокруг С-гликозидной связи между атомами углерода C-(1") sp ${ }^{3}-\mathrm{C}-(6) \mathrm{sp}^{2}$.

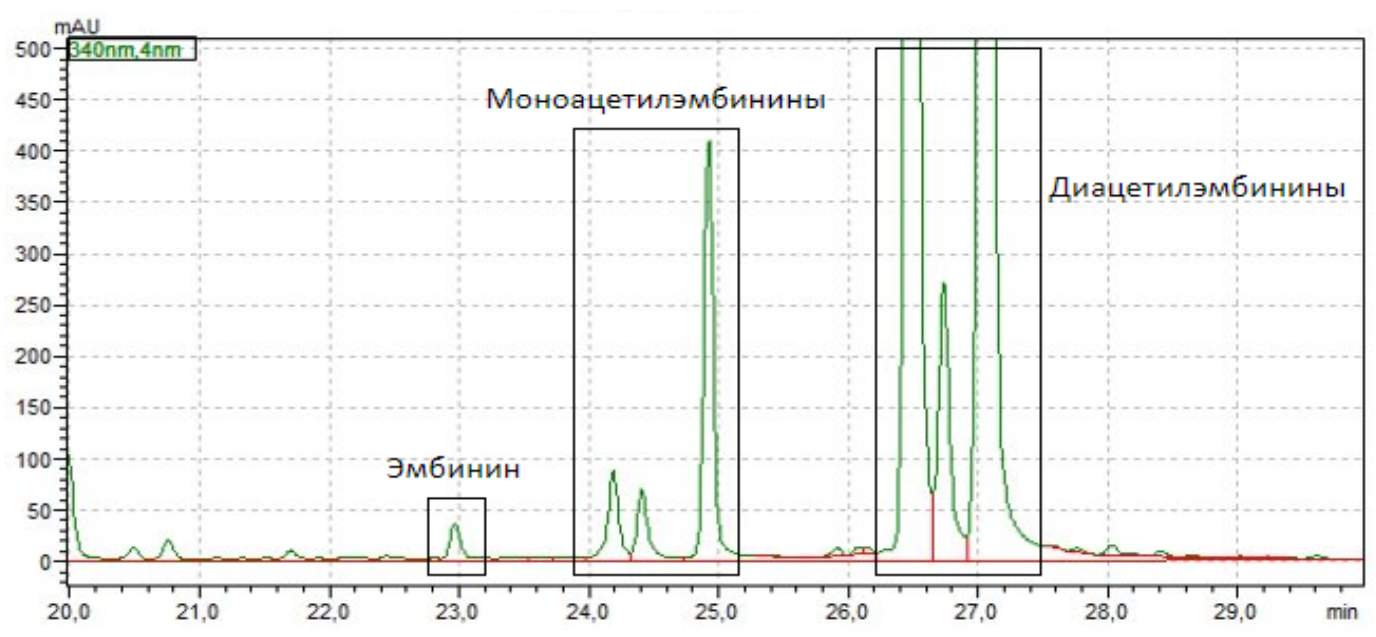

Рис. 1. ВЭЖХ хроматограмма суммы С-гликозидов - эмбинин, три моноацетилэмбинина и три диацетилэмбинина 
Явление дублированных спектральных сигналов из-за одновременного существования двух взаимопревращающихся ротамеров в растворе ранее сообщалось в литературе для других С-гликозидов, либо содержащих стерические препятствия (остатки О-гликозида или О-Ме) в орто- положениях ароматического кольца, прилегающих к С-гликозидной связи, либо имеющих О-гликозид, связанный с самим С-гликозидным остатком [20-22].

При повышении температуры ${ }^{1} \mathrm{H}-Я М Р$ экспериментов дублированные сигналы двух ротамеров сливаются в один усредненный из-за увеличения скорости взаимного превращения ротамеров.

Структуру соединения 7 устанавливали с применением вышеупомянутых ЯМР-методов (табл.). Сигналы ароматических протонов при $\delta_{\mathrm{H}} 8.06(2 \mathrm{H}$, д, J $=8.7$ Гц, Н-2' и Н-6') и 7.08 ( $2 \mathrm{H}$, д, J $=8.7$ Гц, Н-3' и Н-5') свидетельствовали о наличии 4'-замещенного кольца В с АA'BB' спиновой системой. Удвоенные синглеты при $\delta_{\mathrm{H}} 6.92$ и $\delta_{\mathrm{H}} 6.93$ были соотнесены с Н-3 и Н-8 протонам колец С и А соответственно. Дублированные синглеты при $\delta_{\mathrm{H}} 3.89$ и 3.85 , а также синглет при $\delta_{\mathrm{H}} 3.82$ представляли 7-ОМе и 4'-ОМе метокси-группы. Сигналы аномерных протонов при $\delta_{\mathrm{H}} 4.67\left(1 \mathrm{H}\right.$, д, J = 9.7 Гц, Н-1") и $\delta_{\mathrm{H}} 5.23\left(1 \mathrm{H}\right.$, д, J $\left.=1.0 \mathrm{~Hz}, \mathrm{H}-1{ }^{\prime \prime}\right)$ демонстрировали, что соединение 7 представляет собой бигликозид. Основываясь на химических сдвигах аномерных протонов, можно предположить, что один из гликозидных остатков является С-гликозидом, а другой гликозидный остаток является О-гликозидом. Рассматривая аномерную константу спин-спинового взаимодействия (КССВ) равную 1.0 Гц и наличие метильной группы в кольце данного углеводного остатка очевидно, что О-гликозидный остаток представлен $\alpha$-L-рамнопиранозой. COSY корреляции наряду с КCCB были использованы для установления химических сдвигов углеводных протонов, начиная с аномерного протона в обоих кольцах (рис. 2). Полученный спектр DEPT позволил обнаружить 6"-- $\mathrm{CH}_{2} \mathrm{OH}$ группу глюкозного остатка.

Спектр NOESY позволил нам дополнительно подтвердить стереохимию кольца в остатке глюкозы, используя корреляции между аксиальными протонами в положениях Н-1", Н-3" и Н-5" и в остатке рамнозы, используя корреляции между аксиальными протонами в положениях Н-1"' и Н-4"' с протонами группы 6"'$\mathrm{CH}_{3}$. Взаимосвязь остатка рамнозы с остатком глюкозы подтверждено с помощью корреляции NOESY между аномерным протоном рамнозы H-1"' с Н-1", Н-3" и Н-5" протонами глюкозы и НМВС корреляции между аномерным протоном рамнозы Н-1"' с С-2"' глюкозы, располагающим О-гликозидную связь в 2"- по-

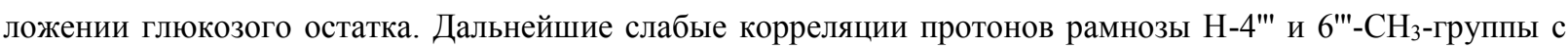
протонами глюкозы в положениях Н-1", Н-3" и Н-5" дополнительно подтвердили расположение $1 \rightarrow 2$ О-гликозидной связи (рис. 3).

Расположение ацетатов в остатке $\alpha$-L-рамнозы определено с использованием того факта, что протоны углеводного кольца, связанные с тем же атомом углерода, что и ацетокси-группа, демонстрируют сильный химический сдвиг в область более слабого поля по сравнению с протонами, присоединенными к углероду с незамещенной гидроксильной группой. В соединении 7 сигналы протонов в положении Н-4"' и Н-3"' сдвинуты в область более слабого поля к $\delta_{\mathrm{H}} 4.52$ и $\delta_{\mathrm{H}} 4.43$, соответственно, что позволило нам предположить, что гидроксильные группы в положениях С-3"' и С-4"' ацетилированы. Соответственно соединение 7 имеет структуру 3"',4"'-диацетилэмбинина.

Спектральные характеристики соединения 5 практически идентичны с соединением 7 (табл.). Основное различие связано с сигналами, полученными от $\alpha$-L-рамнозы, особенно от протонов в положении $\mathrm{H}-2 \mathrm{2}$ и Н-4"', которые были смещены в область более слабого поля $\delta_{\text {н }} 4.84$ и 4.35 , соответственно. В результате чего можно сделать заключение, что гидроксильные группы при C-2"' и С-4"' имеют остатки ацетата, образующие 2"',4"'-диацетилэмбинин.

\section{Ступенчатый гидролиз соединений 5 и 7}

Хроматограммы, полученные для выделенных С-гликозидов (5 и 7), после растворения в среде $0.1 \%$ (об./об.) ТФУ вода - ацетонитрил ( $1: 1)$, показали наличие продуктов ступенчатого гидролиза их ацетатных остатков (рис. 4 и 5). Этот факт наряду со знанием характера ацетилирования в соединениях 5 и 7 после установления их структуры позволил нам установить структуру соединений $\mathbf{2 - 4}$ и $\mathbf{6}$. Оба соединения -2 "', 3 "'диацетилэмбинин (5) и 3"',4"'-диацетилэмбинин (7), имеют ацетатный остаток в 3"'-положении рамнозного кольца, следовательно, 3 "'-ацетилэмбинин является одним из продуктов ступенчатого гидролиза в обоих случаях. Помимо 3 "'-ацетилэмбинина, вторым продуктом гидролиза 2 "', 3 "'-диацетилэмбинина (5) должен быть 2"'-ацетилэмбинин (рис. 4), а 3"',4"'-диацетилэмбинина (7) - 4"'-ацетилэмбинин (рис. 5). Из экспериментов ВЭЖХ-МС известно, что соединение 6 является диацетатным производным эмбинина. При ступенчатом 
гидролизе соединение 6 образовывал 2 "'-ацетилэмбинин и 4"'-ацетилэмбинин в качестве основных продуктов (рис. 6), из чего можно заключить, что соединение 6 является 2"',4"'-диацетилэмбинин. Моноацетилэмбинины (2-4) образовавали эмбинин как единственный продукт своего гидролиза (рис. 7).

Выделенная сумма С-гликозидов (1-7) были дополнительно подвергнуты фрагментации методом ВЭЖХ-МС/МС. Соединения 2-4 проявляли одинаковый характер фрагментации. Соединения 5-7 также проявляли одинаковый характер фрагментации между собой.

${ }^{1} \mathrm{H}$ и ${ }^{13} \mathrm{C}$ данные для соединений $\mathbf{1 , 5}$ и 7

\begin{tabular}{|c|c|c|c|c|c|c|c|c|}
\hline \multirow{3}{*}{$\begin{array}{c}\text { Положе- } \\
\text { ние }\end{array}$} & \multicolumn{4}{|c|}{ Соединение 7} & \multicolumn{2}{|c|}{ Соединение 5} & \multicolumn{2}{|c|}{ Соединение 1} \\
\hline & \multicolumn{2}{|c|}{ Маж. ротамер } & \multicolumn{2}{|c|}{ Мин. роматер } & \multirow{2}{*}{$\begin{array}{c}\text { Маж. ро- } \\
\text { тамер } \\
\delta \text { н }(J \text { in } \\
H z)\end{array}$} & \multirow{2}{*}{$\begin{array}{c}\text { Мин. po- } \\
\text { матер } \\
\delta \text { H }(J \text { in } \\
\mathrm{Hz})\end{array}$} & \multirow{2}{*}{$\begin{array}{c}\text { Маж. po- } \\
\text { тамер } \\
\delta \text { н }(J \text { in } \\
H z)\end{array}$} & \multirow{2}{*}{$\begin{array}{c}\text { Мин. po- } \\
\text { матер } \\
\delta \text { н }(J \text { in } \\
\mathrm{Hz})\end{array}$} \\
\hline & $\begin{array}{c}\delta \mathrm{H}(J \text { in } \\
\mathrm{Hz})\end{array}$ & $\delta \mathrm{C}$ & $\begin{array}{c}\delta \mathrm{H}(J \text { in } \\
\mathrm{Hz})\end{array}$ & $\delta \mathrm{C}$ & & & & \\
\hline $2-\mathrm{C}$ & & 164.14 & & 164.24 & & & & \\
\hline $3-\mathrm{CH}$ & $6.93, \mathrm{~s}$ & 110.74 & $6.89, \mathrm{~s}$ & 110.56 & $6.89, \mathrm{~s}$ & $6.84, \mathrm{~s}$ & $6.87, \mathrm{~s}$ & $6.88, \mathrm{~s}$ \\
\hline $4-\mathrm{C}$ & & 183.09 & & 182.63 & & & & \\
\hline $4 a-C$ & & 104.37 & & 104.56 & & & & \\
\hline $5-\mathrm{C}$ & & 160.89 & & 159.97 & & & & \\
\hline $6-\mathrm{C}$ & & 110.74 & & 110.56 & & & & \\
\hline $7-\mathrm{C}$ & & 165.53 & & 164.35 & & & & \\
\hline $8-\mathrm{CH}$ & $6.92, \mathrm{~s}$ & 105.51 & $6.88, \mathrm{~s}$ & 105.22 & $6.92, \mathrm{~s}$ & $6.85, \mathrm{~s}$ & $6.94, \mathrm{~s}$ & $6.92, \mathrm{~s}$ \\
\hline $8 \mathrm{a}-\mathrm{C}$ & & 157.82 & & 157.90 & & & & \\
\hline 1'-C & & 123.15 & & 123.30 & & & & \\
\hline 2',6'-CH & $\begin{array}{c}8.06, \mathrm{~d} \\
(8.7)\end{array}$ & 129.20 & $\begin{array}{c}8.03, \mathrm{~d} \\
(8.7)\end{array}$ & 129.20 & $\begin{array}{c}8.04, \mathrm{~d} \\
(9.0)\end{array}$ & $\begin{array}{c}8.04, \mathrm{~d} \\
(9.0)\end{array}$ & $\begin{array}{c}8.08, \mathrm{~d} \\
(8.4)\end{array}$ & $\begin{array}{c}8.07, \mathrm{~d} \\
(8.4)\end{array}$ \\
\hline 3',5'-CH & $\begin{array}{c}7.08, \mathrm{~d} \\
(8.7)\end{array}$ & 115.56 & $\begin{array}{c}7.08, \mathrm{~d} \\
(8.7)\end{array}$ & 115.56 & $\begin{array}{c}7.09, \mathrm{~d} \\
(9.0)\end{array}$ & $\begin{array}{c}7.09, \mathrm{~d} \\
(9,0)\end{array}$ & $\begin{array}{l}\text { 7. } 11, \mathrm{~d} \\
(8.7)\end{array}$ & $\begin{array}{c}7.11, \mathrm{~d} \\
(8.7)\end{array}$ \\
\hline $4^{\prime}-\mathrm{C}$ & & 163.33 & & 163.33 & & & & \\
\hline 1"-CH & $\begin{array}{c}4.67, \mathrm{~d} \\
(9.7)\end{array}$ & 73.04 & $\begin{array}{c}4.67, \mathrm{~d} \\
(9.7)\end{array}$ & 72.04 & $\begin{array}{l}4.63, \mathrm{~d} \\
(9.4)\end{array}$ & $\begin{array}{l}4.63, \mathrm{~d} \\
(9.4)\end{array}$ & $4.63, \mathrm{~m}$ & $4.63, \mathrm{~m}$ \\
\hline 2"-CH & $\begin{array}{c}4.23, \mathrm{dd} \\
(9.7,9.3)\end{array}$ & 72.59 & $\begin{array}{c}4.06, \mathrm{dd} \\
(9.7,9.3)\end{array}$ & 72.39 & $\begin{array}{c}4.23, \mathrm{dd} \\
(9.6,9.0)\end{array}$ & $\begin{array}{c}4.03, \mathrm{dd} \\
(9.7,9.3)\end{array}$ & $4.31, \mathrm{~m}$ & $4.14, \mathrm{~m}$ \\
\hline 3"-CH & $3.42, \mathrm{~m}$ & 77.48 & $3.42, \mathrm{~m}$ & 77.48 & $3.37, \mathrm{~m}$ & $3.37, \mathrm{~m}$ & $3.35, \mathrm{~m}$ & $3.33, \mathrm{~m}$ \\
\hline 4"-CH & $3.11, \mathrm{~m}$ & 71.77 & $3.10, \mathrm{~m}$ & 71.77 & $3.10 \mathrm{~m}$ & $3.10, \mathrm{~m}$ & $3.10, \mathrm{~m}$ & $3.10, \mathrm{~m}$ \\
\hline 5"-CH & $3.12, \mathrm{~m}$ & 80.70 & $3.12, \mathrm{~m}$ & 80.59 & $3.09, \mathrm{~m}$ & $3.09, \mathrm{~m}$ & $3.09, \mathrm{~m}$ & $3.09, \mathrm{~m}$ \\
\hline 6"--CH & $3.65, \mathrm{~m}$ & 62.26 & $3.65, \mathrm{~m}$ & 61.82 & $3.57, \mathrm{~m}$ & $3.57, \mathrm{~m}$ & $3.60, \mathrm{~m}$ & $3.60, \mathrm{~m}$ \\
\hline 1"'-CH & $\begin{array}{c}5.23, \mathrm{~d} \\
(1.0)\end{array}$ & 99.60 & $\begin{array}{c}5.10, \mathrm{~d} \\
(1.0)\end{array}$ & 100.30 & $\begin{array}{c}5.14, \mathrm{~d} \\
(1.0)\end{array}$ & $\begin{array}{c}5.18, \mathrm{~d} \\
(1.4)\end{array}$ & 5.03, brs & 4.93 , brs \\
\hline 2"'-CH & $3.67, \mathrm{~m}$ & 71.32 & $3.67, \mathrm{~m}$ & 71.28 & $\begin{array}{l}4.84, \mathrm{dd} \\
(8.6,1.8)\end{array}$ & $\begin{array}{l}4.83, \mathrm{dd} \\
(8.6,1.8)\end{array}$ & $3.58, \mathrm{~m}$ & $3.58, \mathrm{~m}$ \\
\hline 3"'-CH & $\begin{array}{c}4.43, \mathrm{dd} \\
(9.9,3.0)\end{array}$ & 71.75 & $\begin{array}{c}4.39 \mathrm{dd} \\
(9.9,3.0)\end{array}$ & 71.24 & $3.42, \mathrm{~m}$ & $3.42, \mathrm{~m}$ & $3.07, \mathrm{~m}$ & $3.07, \mathrm{~m}$ \\
\hline 4"'-CH & $\begin{array}{c}4.52, \mathrm{dd} \\
(9.9,9.5)\end{array}$ & 73.45 & $\begin{array}{c}4.52, \mathrm{dd} \\
(9.9,9.5)\end{array}$ & 73.40 & $4.35, \mathrm{~m}$ & $4.38, \mathrm{~m}$ & $2.90, \mathrm{~m}$ & $2.85, \mathrm{~m}$ \\
\hline 5"'-CH & $2.19, \mathrm{~m}$ & 66.07 & $2.19, \mathrm{~m}$ & 65.93 & $2.12, \mathrm{~m}$ & $2,12 \mathrm{~m}$ & $2.11, \mathrm{~m}$ & $2.25, \mathrm{~m}$ \\
\hline 6"'--CH3 & $\begin{array}{c}0.43, \mathrm{~d} \\
(6.3)\end{array}$ & 17.74 & $\begin{array}{c}0.47, \mathrm{~d} \\
(6.3)\end{array}$ & 18.40 & $\begin{array}{c}0.35, \mathrm{~d} \\
(6.3)\end{array}$ & $\begin{array}{c}0.43, \mathrm{~d} \\
(6.3)\end{array}$ & $0.44, \mathrm{~m}$ & $0.56, \mathrm{~m}$ \\
\hline $5-\mathrm{OH}$ & $13.37, \mathrm{~s}$ & - & $13.41, \mathrm{~s}$ & - & $13.40, \mathrm{~s}$ & $13.38, \mathrm{~s}$ & $13.39, \mathrm{~s}$ & $13.39, \mathrm{~s}$ \\
\hline 7-OMe & $3.89, \mathrm{~s}$ & 57.50 & $3.85, \mathrm{~s}$ & 57.35 & $3.90, \mathrm{~s}$ & $3.85, \mathrm{~s}$ & $3.89, \mathrm{~s}$ & $3.87, \mathrm{~s}$ \\
\hline 4'-OMe & $3.82, \mathrm{~s}$ & 56.57 & $3.82, \mathrm{~s}$ & 56.57 & $3.81, \mathrm{~s}$ & $3.81, \mathrm{~s}$ & $3.85, \mathrm{~s}$ & $3.85, \mathrm{~s}$ \\
\hline 2"'-OAc & - & & - & & $1.90, \mathrm{~s}$ & $1.93, \mathrm{~s}$ & & \\
\hline 3"'-OAc & $1.88, \mathrm{~s}$ & $\begin{array}{c}170.61 \\
21.15\end{array}$ & $1.86, \mathrm{~s}$ & $\begin{array}{l}170.45 \\
21.18\end{array}$ & - & - & & \\
\hline 4"'-OAc & $1.65, \mathrm{~s}$ & $\begin{array}{c}170.12 \\
20.38\end{array}$ & $1.60, \mathrm{~s}$ & $\begin{array}{c}170.12 \\
20.33\end{array}$ & $1.59, \mathrm{~s}$ & $1.60, \mathrm{~s}$ & & \\
\hline
\end{tabular}




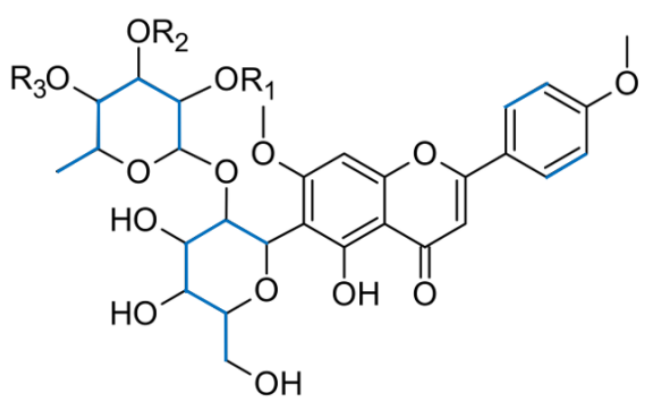

Рис. 2. Ключевые ${ }^{1} \mathrm{H}-{ }^{1} \mathrm{H}$ COSY корреляции характерные для соединений $\mathbf{1}, \mathbf{5}$ и $\mathbf{7}$ (синий)

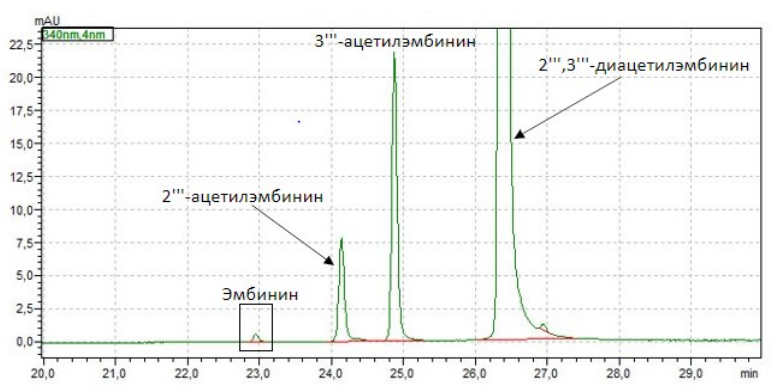

Рис. 4. Хроматограмма после ступенчатого гидролиза 2"',3"'-диацетилэмбинина (5)

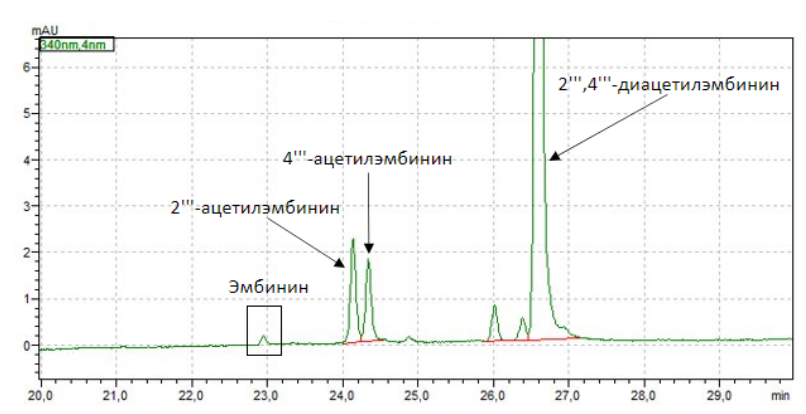

Рис. 6. Хроматограмма после ступенчатого гидролиза 2"',4"'-диацетилэмбинина (6)

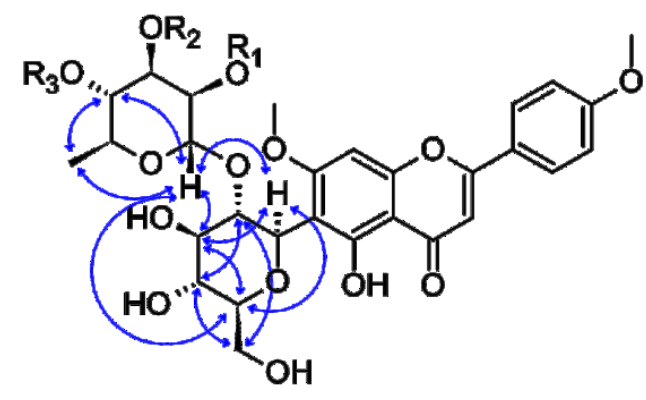

Рис. 3. Ключевые ${ }^{1} \mathrm{H}-{ }^{1} \mathrm{H}$ NOESY корреляции, характерные для соединений 5 и 7

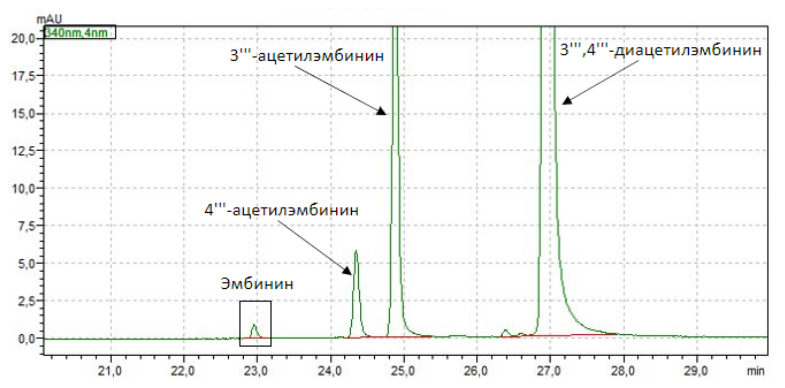

Рис. 5. Хроматограмма после ступенчатого гидролиза 3"',4"'-диацетилэмбинина (7)

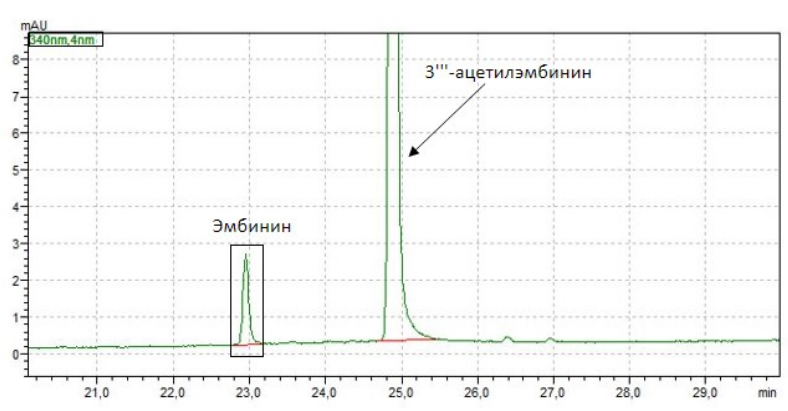

Рис. 7. Хроматограмма после ступенчатого гидролиза 3"'-ацетилэмбинина (3)

\section{Bыводы}

1. В результате проведенных исследований из листьев $R$. chamaemorus впервые для представителей рода Rubus идентифицированы семь С-гликозидов, а именно:

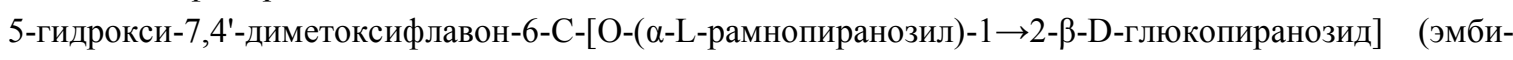
нин) (1),

5-гидрокси-7,4'-диметоксифлавон-6-C-[O-( $\alpha$-L-2"'-ацетилрамнопиранозил)-1 $\rightarrow 2-\beta$-D-глюкопиранозид] (2"'-ацетилэмбинин) (2),

5 -гидрокси-7,4'-диметоксифлавон-6-C-[O-( $\alpha$-L-3"'-ацетилрамнопиранозил)-1 $\rightarrow 2-\beta$-D-глюкопиранозид] (3"'- ацетилэмбинин) (3),

5 -гидрокси-7,4'-диметоксифлавон-6-C-[O-( $\alpha$-L-4"'-ацетилрамнопиранозил)-1 $\rightarrow 2-\beta$-D-глюкопиранозид] (4"'-ацетилэмбинин) (4),

5-гидрокси-7,4'-диметоксифлавон-6-C-[O-( $\alpha$-L-2"',3"'-диацетилрамнопиранозил)-1 $\rightarrow 2-\beta$-D-глюкопиранозид] (2"',3"'-диацетилэмбинин) (5), 


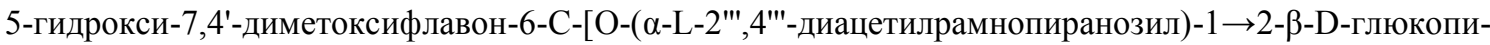
ранозид] (2"',4"' -диацетилэмбинин) (6),

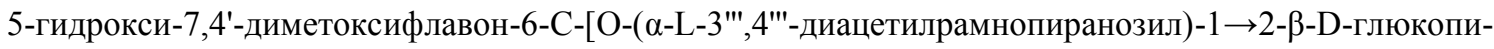
ранозид] (3"',4"'-диацетилэмбинин) (7).

2. С использованием комплекса спектральных методов установлена структура индивидуально выделенных трех С-гликозидов флавонов - эмбинин (1), 2"',3"'-диацетилэмбинин (5) и 3"',4"'-диацетилэмбинин (7).

3. Установлена структура четырех С-гликозидов флавонов $(\mathbf{2}, \mathbf{3}, \mathbf{4 , 6})$ на основании сравнительного хроматографического анализа продуктов их частичного гидролиза с продуктами частичного гидролиза ацетатных остатков соединений 5 и 7.

4. Обнаружено, что моноацетатные и диацетатные производные эмбинина в водно-ацетонитрильном растворе, с нейтральным или слабокислым $\mathrm{pH}$, при комнатной температуре или слабом нагревании, гидролизуются с образованием производных, содержащих на один и / или два ацетатных остатка меньше, чем исходное ацелированное производное С-гликозида. При наличии одинаковых продуктов эффект ступенчатого гидролиза ацетатных остатков неизвестных соединений в сравнении с данными ступенчатого гидролиза ацетатных остатков известных соединений может использоваться для установления структуры С-гликозилированных соединений.

5. С-гликозиды - эмбинин и его ацетатные производные являются редкими вторичными метаболитами высших растений, принадлежащими к разным семействам и произрастающим в сильно различающихся средах обитания, что может указывать на еще неизвестные сходные функции этих групп соединений в физиологии и биохимии растений.

6. Листья $R$. chamaemorus являются перспективным источником соединений, обладающих иммунотропным и кардиотоническим потенциалом.

\section{Список литературы}

1. Taylor K. Biological flora of the British Isles. Rubus chamaemorus L. // Journal of Ecology. 1971. Vol. 59. Pp. 293306.

2. Lohi K. Variation between cloudberries (Rubus chamaemorus L.) in different habitats // Aquilo Ser. Botanica. 1974. Vol. 13. P. 19.

3. Korpelainen H. Sex rations and resource allocation among sexually reproducing plants of Rubus chamaemorus // Annals of Botany. 1994. Vol. 74. Pp. 627-632. DOI: 10.1006/anbo.1994.1164.

4. Barbara T. Rubus chamaemorus L. - a boreal plant rich in biologically active metabolites: A review // Biology Letters. 2003. Vol. 40. N1. Pp. 3-13.

5. McDougall G.J., Martinussen I., Junttila O., Verrall S., Stewart D. Assessing the Influence of Genotype and Temperature on Polyphenol Composition in Cloudberry (Rubus chamaemorusL.) Using a Novel Mass Spectrometric Method // Journal of Agricultural and Food Chemistry. 2011. Vol. 59. N20. Pp. 10860-10868. DOI: 10.1021/jf202083b.

6. Kähkönen M.P., Hopia A.I., Heinonen M. Berry Phenolics and Their Antioxidant Activity // Journal of Agricultural and Food Chemistry. 2001. Vol. 49. N8. Pp. 4076-4082. DOI: 10.1021/jf010152t.

7. Kähkönen M., Kylli P., Ollilainen V., Salminen J., Heinonen M. Antioxidant Activity of Isolated Ellagitannins from Red Raspberries and Cloudberries // Journal of agricultural and food chemistry. 2012. Vol. 60. N5. Pp. 1167-1174. DOI: $10.1021 / \mathrm{jf} 203431 \mathrm{~g}$.

8. Kaisu M., Afaf K., Törrönen A.R. Identification and Quantification of Phenolic Compounds in Berries of Fragaria and Rubus Species (Family Rosaceae) // Journal of Agricultural and Food Chemistry. 2004. Vol. 52. N20. Pp. 6178-6187. DOI: $10.1021 /$ jf049450r.

9. Määttä-Riihinen K.R., Kamal-Eldin A., Törrönen A.R. Identification and Quantification of Phenolic Compounds in Berries of Fragaria and Rubus Species (Family Rosaceae) // Journal of Agricultural and Food Chemistry. 2004. Vol. 52. N20. Pp. 6178-6187. DOI: 10.1021/jf049450r.

10. Häkkinen S.H., Kärenlampi S.O., Heinonen I.M., Mykkänen H.M., Törrönen A.R. Content of the Flavonols Quercetin, Myricetin, and Kaempferol in 25 Edible Berries // Journal of Agricultural and Food Chemistry. 1999. Vol. 47. N6. Pp. 2274-2279. DOI: $10.1021 /$ jf9811065.

11. Уэйли А.К., Понкратова А.О., Теслов Л.С., Лужанин В.Г. Обзор вторичных метаболитов морошки и их биологической активности // Пульс. 2020. Т. 22. №7. С. 50-59.

12. Whaley A.K., Ebrahim W., El-Neketi M., Ancheeva E.U., Özkaya F.C., Pryakhina N.I., Sipkina N.U., Luzhanin V.G., Liu Z., Proksch P. New acetylated flavone C -glycosides from Iris lactea // Tetrahedron Letters. 2017. Vol. 58. N22. Pp. 2171-2173. DOI:10.1016/j.tetlet.2017.04.080.

13. Pryakhina N.I., Sheichenko V.I., Blinova K.F. Acylated C-glycosides of Iris lactea // Chemistry of Natural Compounds. 1984. Vol. 20. N5. Pp. 554-559. 
14. Shen W.J., Qin M.J., Shu P., Zang C.F. Two new C-glycosylflavones from the leaves of Iris lactea var. chinensis // Chinese Chemical Letters. 2008. Vol. 19. N7. Pp. 821-824. DOI: 10.1016/j.cclet.2008.04.031.

15. Kawase A., Yagishita K. On the Structure of a New C-Glycosyl Flavone, Embinin, Isolated from the Petals of Iris germanica L. // Agricultural and Biological Chemistry. 1968. Vol. 32. N4. Pp. 537-538. DOI: 10.1080/00021369.1968.10859095.

16. Meng Y., Qin M., Qi B., Xie G. Four new C-glycosylflavones from the leaves of Iris lactea Pall. var. chinensis (Fisch.) Koidz. // Phytochemistry Letters. 2017. Vol. 22. Pp. 33-38. DOI: 10.1016/j.phytol.2017.08.011.

17. Shen W.J., Qin M.J., Shu P., Zhang C.F. Two new C-glycosylflavones from the leaves of Iris lactea var. chinensis // Chinese Chemical Letters. 2008. Vol. 19. N7. Pp. 821-824. DOI: 10.1016/j.cclet.2008.04.031.

18. Бычкова Н.В., Калашникова А.А., Уэйли А.К., Лужанин В.Г., Калинина Н.М., Шустов Е.Б., Оковитый С.В. Изучение иммунотропных эффектов флавоноидного гликозида эмбинина методом проточной цитометрии // Профилактическая и клиническая медицина. 2019. Т. 4. №73. С. 77-82.

19. Ивкин Д.Ю., Лужанин В.Г., Карпов А.А., Минасян С.М., Полещенко Я.И., Мамедов А.Э., Повыдыш М.Н., Поройков В.В., Наркевич И.А. Эмбинин - перспективное кардиотоническое средство природного происхождения // Разработка и регистрация лекарственных средств. 2018. Т. 3. №24. С. 166-170.

20. Rayyan S., Fossen T., Nateland H.S., Andersen Ø.M. Isolation and identification of flavonoids, including flavone rotamers, from the herbal drug "crataegi folium cum flore" (hawthorn) // Phytochemical Analysis. 2005. Vol. 16. N5. Pp. 334-341. DOI: 10.1002/pca.853.

21. Zhou G., Yan R., Wang X., Li S., Lin J., Liu J., Zhao Z. The overlooked rotational isomerism of C-glycosyl flavonoids // Phytochemistry Reviews. 2019. Vol. 18. N2. Pp. 443-461. DOI: 10.1007/s11101-019-09601-7.

22. Lewis K., Maxwell A., McLean S., Reynolds W., Enriquez R. Room - temperature $\left({ }^{1} \mathrm{H},{ }^{13} \mathrm{C}\right)$ and variable - temperature $\left({ }^{1} \mathrm{H}\right)$ NMR studies on spinosin // Magnetic Resonance in Chemistry. 2000. Vol. 38. Pp. 771-774. DOI: 10.1002/1097458X(200009)38:9<771::AID-MRC729>3.0.CO;2-4.

Поступила в редакиию 20 января 2021 г.

После переработки 4 февраля 2021 г.

Принята к публикации 3 марта 2021 г.

Для цитирования: Уэйли А.К., Понкратова А.О., Орлова А.А., Серебряков Е.Б., Селиванов С.И., Кривощеков С.В., Белоусов М.В., Прокш П., Лужанин В.Г. Анализ С-гликозидов флавонов и продуктов ступенчатого гидролиза их ацетатов в листьях Rubus chamaemorus L. // Химия растительного сырья. 2021. №2. С. 257-265. DOI: $10.14258 /$ jcprm. 2021029185 .

Whaley A.K. ${ }^{l}$, Ponkratova A.O..$^{*}$, Orlova A.A. ${ }^{l}$, Serebryakov E.B. ${ }^{2}$, Selivanov S.I. ${ }^{2}$, Krivoshchekov S.V. ${ }^{3}$, Belousov M.V. ${ }^{3}$, Proksch $P{ }^{4}$, Luzhanin V.G. ${ }^{1}$ ANALYSIS OF FLAVONES C-GLYCOSIDES AND AND STEPWISE HYDROLYSIS OF THEIR ACETATES IN THE LEAVES OF RUBUS CHAMAEMORUS L.

${ }^{1}$ Saint Petersburg State Chemical Pharmaceutical University, ul. Prof. Popova 14, Saint Petersburg, 197376 (Russia), e-mail: anastasiya.ponkratova@yandex.ru

${ }_{2}^{2}$ Saint Petersburg State University, Universitetskii pr. 26, Saint Petersburg, 198504 (Russia)

${ }^{3}$ Siberian State Medical University, Moskovsky trakt 2, Tomsk, 634050 (Russia)

${ }^{4}$ Institute of Pharmaceutical Biology and Biotechnology, Heinrich-Heine-Universität Düsseldorf, Universitätsstrasse 1, Düsseldorf, 40225 (Germany)

The C-glycoside embinin and its mono- and diacetate derivatives have immunotropic and cardiotonic activity, which makes the search for plants that contain them interesting. Embinin and its acetate derivatives were previously isolated only from some plants of the genus Iris, the habitat and growing conditions of which are very different from those of the genus Rubus. As a result of the study, the structure of seven C-glycosides, embinin derivatives, isolated from the leaves of Rubus chamaemorus L. (Rosaceae) was established. Using HR-ESI-MS, HPLC-MS, as well as one- and two-dimensional NMR spectroscopy, the structure of three substances isolated in individual form was established: embinin (1) and its diacetyl derivatives - 2"',3'"-diacetylembinin (5) and 3"',4"'-diacetylembinin (7). The method of stepwise hydrolysis of C-glycoside acetate residues proposed in this study, followed by HPLC analysis of the resulting hydrolysis products, made it possible to establish the structure of minor flavone C-glycosides contained in the leaves of Rubus chamaemorus L.: 2"'-acetylembinin (2), 3"'-acetylembinin (3), 4"'-acetylembinin (4) and 2"',4"'-diacetylembinin (6). All these compounds were found in the leaves of Rubus chamaemorus L. for the first time. The C-glycosides - embinin and its acetate derivatives are rare metabolites of higher plants, the presence of which is determined by the peculiarity of their physiology, and the biological activity determines the prospects for medical use.

Keywords: C-glycosides, flavones, hydrolysis, Rubus chamaemorus, secondary metabolites.

\footnotetext{
${ }^{*}$ Corresponding author.
} 


\section{References}

1. Taylor K. Journal of Ecology, 1971, vol. 59, pp. 293-306.

2. Lohi K. Aquilo Ser. Botanica, 1974, vol. 13, p. 19.

3. Korpelainen H. Annals of Botany, 1994, vol. 74, pp. 627-632. DOI: 10.1006/anbo.1994.1164.

4. Barbara T. Biology Letters, 2003, vol. 40, no. 1, pp. 3-13.

5. McDougall G.J., Martinussen I., Junttila O., Verrall S., Stewart D. Journal of Agricultural and Food Chemistry, 2011, vol. 59, no. 20, pp. 10860-10868. DOI: 10.1021/jf202083b.

6. Kähkönen M.P., Hopia A.I., Heinonen M. Journal of Agricultural and Food Chemistry, 2001, vol. 49, no. 8, pp. 40764082. DOI: $10.1021 / \mathrm{jf0} 10152 \mathrm{t}$.

7. Kähkönen M., Kylli P., Ollilainen V., Salminen J., Heinonen M. Journal of agricultural and food chemistry, 2012, vol. 60, no. 5, pp. 1167-1174. DOI: 10.1021/jf203431g.

8. Kaisu M., Afaf K., Törrönen A.R. Journal of Agricultural and Food Chemistry, 2004, vol. 52, no. 20, pp. 6178-6187. DOI: $10.1021 / \mathrm{jf049450r.}$

9. Määttä-Riihinen K.R., Kamal-Eldin A., Törrönen A.R. Journal of Agricultural and Food Chemistry, 2004, vol. 52, no. 20, pp. 6178-6187. DOI: 10.1021/jf049450r.

10. Häkkinen S.H., Kärenlampi S.O., Heinonen I.M., Mykkänen H.M., Törrönen A.R. Journal of Agricultural and Food Chemistry, 1999, vol. 47, no. 6, pp. 2274-2279. DOI: 10.1021/jf9811065.

11. Ueyli A.K., Ponkratova A.O., Teslov L.S., Luzhanin V.G. Pul's, 2020, vol. 22, no. 7, pp. 50-59. (in Russ.).

12. Whaley A.K., Ebrahim W., El-Neketi M., Ancheeva E.U., Özkaya F.C., Pryakhina N.I., Sipkina N.U., Luzhanin V.G., Liu Z., Proksch P. Tetrahedron Letters, 2017, vol. 58, no. 22, pp. 2171-2173. DOI:10.1016/j.tetlet.2017.04.080.

13. Pryakhina N.I., Sheichenko V.I., Blinova K.F. Chemistry of Natural Compounds, 1984, vol. 20, no. 5, pp. 554-559.

14. Shen W.J., Qin M.J., Shu P., Zang C.F. Chinese Chemical Letters, 2008, vol. 19, no. 7, pp. 821-824. DOI: 10.1016/j.cclet.2008.04.031.

15. Kawase A., Yagishita K. Agricultural and Biological Chemistry, 1968, vol. 32, no. 4, pp. 537-538. DOI: 10.1080/00021369.1968.10859095.

16. Meng Y., Qin M., Qi B., Xie G. Phytochemistry Letters, 2017, vol. 22, pp. 33-38. DOI: 10.1016/j.phytol.2017.08.011.

17. Shen W.J., Qin M.J., Shu P., Zhang C.F. Chinese Chemical Letters, 2008, vol. 19, no. 7, pp. 821-824. DOI: 10.1016/j.cclet.2008.04.031.

18. Bychkova N.V., Kalashnikova A.A., Ueyli A.K., Luzhanin V.G., Kalinina N.M., Shustov Ye.B., Okovityy S.V. Profilakticheskaya i klinicheskaya meditsina, 2019, vol. 4, no. 73, pp. 77-82. (in Russ.).

19. Ivkin D.Yu., Luzhanin V.G., Karpov A.A., Minasyan S.M., Poleshchenko Ya.I., Mamedov A.E., Povydysh M.N., Poroykov V.V., Narkevich I.A. Razrabotka i registratsiya lekarstvennykh sredstv, 2018, vol. 3, no. 24, pp. 166-170. (in Russ.).

20. Rayyan S., Fossen T., Nateland H.S., Andersen Ø.M. Phytochemical Analysis, 2005, vol. 16, no. 5, pp. 334-341. DOI: 10.1002/pca.853.

21. Zhou G., Yan R., Wang X., Li S., Lin J., Liu J., Zhao Z. Phytochemistry Reviews, 2019, vol. 18, no. 2, pp. $443-461$. DOI: 10.1007/s11101-019-09601-7.

22. Lewis K., Maxwell A., McLean S., Reynolds W., Enriquez R. Magnetic Resonance in Chemistry, 2000, vol. 38, pp. 771-774. DOI: 10.1002/1097-458X(200009)38:9<771::AID-MRC729>3.0.CO;2-4.

Received January 20, 2021

Revised February 4, 2021

Accepted March 3, 2021

For citing: Whaley A.K., Ponkratova A.O., Orlova A.A., Serebryakov E.B., Selivanov S.I., Krivoshchekov S.V., Belousov M.V., Proksch P., Luzhanin V.G. Khimiya Rastitel'nogo Syr'ya, 2021, no. 2, pp. 257-265. (in Russ.). DOI: $10.14258 /$ jcprm.2021029185. 
